\title{
Bounds for the Combinations of Neuman-Sándor, Arithmetic, and Second Seiffert Means in terms of Contraharmonic Mean
}

\author{
Zai-Yin He, ${ }^{1}$ Wei-Mao Qian, ${ }^{2}$ Yun-Liang Jiang, ${ }^{3}$ Ying-Qing Song, ${ }^{4}$ and Yu-Ming Chu ${ }^{4}$ \\ ${ }^{1}$ Department of Mathematics, Huzhou Teachers College, Huzhou 313000, China \\ ${ }^{2}$ School of Distance Education, Huzhou Broadcast and TV University, Huzhou 313000, China \\ ${ }^{3}$ School of Information and Engineering, Huzhou Teachers College, Huzhou 313000, China \\ ${ }^{4}$ School of Mathematics and Computation Science, Hunan City University, \\ Yiyang 413000, China
}

Correspondence should be addressed to Yu-Ming Chu; chuyuming2005@yahoo.com.cn

Received 4 January 2013; Accepted 27 February 2013

Academic Editor: Salvatore A. Marano

Copyright (C) 2013 Zai-Yin He et al. This is an open access article distributed under the Creative Commons Attribution License, which permits unrestricted use, distribution, and reproduction in any medium, provided the original work is properly cited.

We give the greatest values $r_{1}, r_{2}$ and the least values $s_{1}, s_{2}$ in $(1 / 2,1)$ such that the double inequalities $C\left(r_{1} a+\left(1-r_{1}\right) b, r_{1} b+\left(1-r_{1}\right) a\right)<$ $\alpha A(a, b)+(1-\alpha) T(a, b)<C\left(s_{1} a+\left(1-s_{1}\right) b, s_{1} b+\left(1-s_{1}\right) a\right)$ and $C\left(r_{2} a+\left(1-r_{2}\right) b, r_{2} b+\left(1-r_{2}\right) a\right)<\alpha A(a, b)+(1-\alpha) M(a, b)<$ $C\left(s_{2} a+\left(1-s_{2}\right) b, s_{2} b+\left(1-s_{2}\right) a\right)$ hold for any $\alpha \in(0,1)$ and all $a, b>0$ with $a \neq b$, where $A(a, b), M(a, b), C(a, b)$, and $T(a, b)$ are the arithmetic, Neuman-Sándor, contraharmonic, and second Seiffert means of $a$ and $b$, respectively.

\section{Introduction}

For $a, b>0$ with $a \neq b$, the Neuman-Sándor mean $M(a, b)$ [1], second Seiffert mean $T(a, b)[2]$ are defined by

$$
\begin{aligned}
M(a, b) & =\frac{a-b}{2 \sinh ^{-1}((a-b) /(a+b))}, \\
T(a, b) & =\frac{a-b}{2 \arctan ((a-b) /(a+b))},
\end{aligned}
$$

respectively. Herein, $\sinh ^{-1}(x)=\log \left(x+\sqrt{1+x^{2}}\right)$ is the inverse hyperbolic sine function.

Let $H(a, b)=2 a b /(a+b), G(a, b)=\sqrt{a b}, L(a, b)=$ $(a-b) /(\log a-\log b), P(a, b)=(a-b) /[4 \arctan (\sqrt{a / b})-\pi]$ $I(a, b)=1 / e\left(b^{b} / a^{a}\right)^{1 /(b-a)}, A(a, b)=(a+b) / 2, Q(a, b)=$ $\sqrt{\left(a^{2}+b^{2}\right) / 2}$, and $C(a, b)=\left(a^{2}+b^{2}\right) /(a+b)$ be the harmonic, geometric, logarithmic, first Seiffert, identric, arithmetic, quadratic, and contraharmonic means of two distinct positive real numbers $a$ and $b$, respectively. Then it is well known that the inequalities

$$
\begin{aligned}
H(a, b) & <G(a, b)<L(a, b)<P(a, b) \\
& <I(a, b)<A(a, b)<M(a, b) \\
& <T(a, b)<Q(a, b)<C(a, b)
\end{aligned}
$$

hold for all $a, b>0$ with $a \neq b$.

Among means of two variables, the Neuman-Sándor, contraharmonic, and second Seiffert means have attracted the attention of several researchers. In particular, many remarkable inequalities and applications for these means can be found in the literature [3-15].

Neuman and Sándor $[1,16]$ proved that the inequalities

$$
\begin{gathered}
A(a, b)<M(a, b)<\frac{A(a, b)}{\log (1+\sqrt{2})}, \\
\frac{\pi}{4} T(a, b)<M(a, b)<T(a, b), \\
M(a, b)<\frac{2 A(a, b)+Q(a, b)}{3}, \\
P(a, b) M(a, b)<A^{2}(a, b),
\end{gathered}
$$




$$
\begin{aligned}
A(a, b) T(a, b) & <M^{2}(a, b) \\
& <\frac{\left(A^{2}(a, b)+T^{2}(a, b)\right)}{2}
\end{aligned}
$$

hold for all $a, b>0$ with $a \neq b$.

Let $0<a, b<1 / 2$ with $a \neq b, a^{\prime}=1-a$ and $b^{\prime}=1-b$. Then the Ky Fan inequalities

$$
\begin{aligned}
\frac{G(a, b)}{G\left(a^{\prime}, \mathrm{b}^{\prime}\right)} & <\frac{L(a, b)}{L\left(a^{\prime}, b^{\prime}\right)}<\frac{P(a, b)}{P\left(a^{\prime}, b^{\prime}\right)}<\frac{A(a, b)}{A\left(a^{\prime}, b^{\prime}\right)} \\
& <\frac{M(a, b)}{M\left(a^{\prime}, b^{\prime}\right)}<\frac{T(a, b)}{T\left(a^{\prime}, b^{\prime}\right)}
\end{aligned}
$$

can be found in [1].

Li et al. [17] proved that the double inequality $L_{p_{0}}(a, b)<$ $M(a, b)<L_{2}(a, b)$ holds for all $a, b>0$ with $a \neq b$, where $L_{p}(a, b)=\left[\left(b^{p+1}-a^{p+1}\right) /((p+1)(b-a))\right]^{1 / p}(p \neq-1,0)$, $L_{0}(a, b)=I(a, b)$ and $L_{-1}(a, b)=L(a, b)$ is the $p$ th generalized logarithmic mean of $a$ and $b$, and $p_{0}=1.843 \cdots$ is the unique solution of the equation $(p+1)^{1 / p}=2 \log (1+\sqrt{2})$.

In [18], Neuman proved that the inequalities

$$
\begin{gathered}
\alpha Q(a, b)+(1-\alpha) A(a, b)<M(a, b) \\
<\beta Q(a, b)+(1-\beta) A(a, b), \\
\lambda C(a, b)+(1-\lambda) A(a, b)<M(a, b) \\
\quad<\mu C(a, b)+(1-\mu) A(a, b)
\end{gathered}
$$

hold for all $a, b>0$ with $a \neq b$ if and only if $\alpha \leq[1-\log (1+$ $\sqrt{2})] /[(\sqrt{2}-1) \log (1+\sqrt{2})]=0.3249 \cdots, \lambda \leq[1-\log (1+$ $\sqrt{2})] / \log (1+\sqrt{2})=0.1345 \cdots, \beta \geq 1 / 3$ and $\mu \geq 1 / 6$.

Zhao et al. [19] found the least values $\alpha_{1}, \alpha_{2}, \alpha_{3}$ and the greatest values $\beta_{1}, \beta_{2}, \beta_{3}$ such that the double inequalities

$$
\begin{gathered}
\alpha_{1} H(a, b)+\left(1-\alpha_{1}\right) Q(a, b)<M(a, b) \\
<\beta_{1} H(a, b)+\left(1-\beta_{1}\right) Q(a, b), \\
\alpha_{2} G(a, b)+\left(1-\alpha_{2}\right) Q(a, b)<M(a, b) \\
<\beta_{2} G(a, b)+\left(1-\beta_{2}\right) Q(a, b), \\
\alpha_{3} H(a, b)+\left(1-\alpha_{3}\right) C(a, b)<M(a, b) \\
<\beta_{3} H(a, b)+\left(1-\beta_{3}\right) C(a, b)
\end{gathered}
$$

hold for all $a, b>0$ with $a \neq b$.
In $[20,21]$, the authors proved that the double inequalities

$$
\begin{gathered}
\alpha_{1} T(a, b)+\left(1-\alpha_{1}\right) G(a, b)<A(a, b) \\
<\beta_{1} T(a, b)+\left(1-\beta_{1}\right) G(a, b), \\
\alpha_{2} Q(a, b)+\left(1-\alpha_{2}\right) A(a, b)<T(a, b) \\
<\beta_{2} Q(a, b)+\left(1-\beta_{2}\right) A(a, b), \\
Q^{\alpha_{3}}(a, b) A^{1-\alpha_{3}}(a, b)<T(a, b) \\
<Q^{\beta_{3}}(a, b) A^{1-\beta_{3}}(a, b)
\end{gathered}
$$

hold for all $a, b>0$ with $a \neq b$ if and only of $\alpha_{1} \leq 3 / 5, \beta_{1} \geq$ $4 / \pi, \quad \alpha_{2} \leq(4-\pi) /[(\sqrt{2}-1) \pi], \beta_{2} \geq 2 / 3, \alpha_{3} \leq 2 / 3$, and $\beta_{3} \geq 4-2 \log \pi / \log 2$.

For $\alpha, \beta, \lambda, \mu \in(1 / 2,1)$, Chu et al. $[22,23]$ proved that the inequalities

$$
\begin{gathered}
C(\alpha a+(1-\alpha) b, \alpha b+(1-\alpha) a)<T(a, b) \\
<C(\beta a+(1-\beta) b, \beta b+(1-\beta) a), \\
Q(\lambda a+(1-\lambda) b, \lambda b+(1-\lambda) a)<T(a, b) \\
<Q(\mu a+(1-\mu) b, \mu b+(1-\mu) a)
\end{gathered}
$$

hold for all $a, b>0$ with $a \neq b$ if and only if $\alpha \leq(1+$ $\sqrt{4 / \pi-1}) / 2, \beta \geq(3+\sqrt{3}) / 6, \lambda \leq\left(1+\sqrt{16 / \pi^{2}-1}\right) / 2$ and $\mu \geq(3+\sqrt{6}) / 6$.

The aim of this paper is to find the greatest values $r_{1}, r_{2}$ and the least values $s_{1}, s_{2}$ such that the double inequalities

$$
\begin{aligned}
& C\left(r_{1} a+\left(1-r_{1}\right) b, r_{1} b+\left(1-r_{1}\right) a\right) \\
& \quad<\alpha A(a, b)+(1-\alpha) T(a, b) \\
& \quad<C\left(s_{1} a+\left(1-s_{1}\right) b, s_{1} b+\left(1-s_{1}\right) a\right), \\
& C\left(r_{2} a+\left(1-r_{2}\right) b, r_{2} b+\left(1-r_{2}\right) a\right) \\
& \quad<\alpha A(a, b)+(1-\alpha) M(a, b) \\
& \quad<C\left(s_{2} a+\left(1-s_{2}\right) b, s_{2} b+\left(1-s_{2}\right) a\right)
\end{aligned}
$$

hold for any $\alpha \in(0,1)$ and all $a, b>0$ with $a \neq b$.

\section{Lemmas}

In order to prove our main results, we need three lemmas, which we present in this section.

Lemma 1 (see [24, Theorem 1.25]). For $-\infty<a<b<+\infty$, let $f, g:[a, b] \rightarrow \mathbb{R}$ be continuous on $[a, b]$ and differentiable on $(a, b)$, let $g^{\prime}(x) \neq 0$ on $(a, b)$. If $f^{\prime}(x) / g^{\prime}(x)$ is increasing (decreasing) on $(a, b)$, then so are

$$
\frac{f(x)-f(a)}{g(x)-g(a)}, \quad \frac{f(x)-f(b)}{g(x)-g(b)} .
$$


If $f^{\prime}(x) / g^{\prime}(x)$ is strictly monotone, then the monotonicity in the conclusion is also strict.

Lemma 2. Let $u, \alpha \in(0,1)$ and

$$
f_{u, \alpha}(x)=u x^{2}-(1-\alpha)\left(\frac{x}{\arctan x}-1\right) .
$$

Then $f_{u, \alpha}(x)>0$ for all $x \in(0,1)$ if and only if $u \geq(1-\alpha) / 3$ and $f_{u, \alpha}(x)<0$ for all $x \in(0,1)$ if and only if $u \leq(1-\alpha)(4 / \pi-$ $1)$.

Proof. From (12), one has

$$
\begin{gathered}
f_{u, \alpha}\left(0^{+}\right)=0 \\
f_{u, \alpha}\left(1^{-}\right)=u-(1-\alpha)\left(\frac{4}{\pi}-1\right), \\
f_{u, \alpha}^{\prime}(x)=2 x\left[u-\frac{1-\alpha}{2} g(x)\right],
\end{gathered}
$$

where

$$
g(x)=\frac{\left(1+x^{2}\right) \arctan x-x}{x\left(1+x^{2}\right)(\arctan x)^{2}}
$$
then

Let $g_{1}(x)=\arctan x-x /\left(1+x^{2}\right)$ and $g_{2}(x)=x(\arctan x)^{2}$,

$$
\begin{aligned}
& g(x)=\frac{g_{1}(x)}{g_{2}(x)}, \quad g_{1}(0)=g_{2}(0)=0 \\
& \frac{g_{1}^{\prime}(x)}{g_{2}^{\prime}(x)} \\
& =\frac{2 x^{2}}{2 x\left(1+x^{2}\right) \arctan x+\left(1+x^{2}\right)^{2}(\arctan x)^{2}} \\
& =\frac{1}{\left(\left(1+x^{2}\right) \arctan x / x\right)+(1 / 2)\left[\left(1+x^{2}\right) \arctan x / x\right]^{2}} .
\end{aligned}
$$

It is not difficult to verify that the function $\left(1+x^{2}\right)$ $\arctan x / x$ is strictly increasing on $(0,1)$. Then (17) and (18) together with Lemma 1 lead to the conclusion that $g(x)$ is strictly decreasing on $(0,1)$. Moreover, making use of L'Hôpital's rule, we get

$$
\begin{gathered}
g\left(0^{+}\right)=\frac{2}{3} \\
g\left(1^{-}\right)=\frac{4(\pi-2)}{\pi^{2}} .
\end{gathered}
$$

We divide the proof into four cases.

Case 1. $u \geq(1-\alpha) / 3$. Then from (15) and (19) together with the monotonicity of $g(x)$, we clearly see that $f_{u, \alpha}(x)$ is strictly increasing on $(0,1)$. Therefore, $f_{u, \alpha}(x)>0$ for all $x \in(0,1)$ follows from (13) and the monotonicity of $f_{u, \alpha}(x)$.

Case 2. $u \leq 2(1-\alpha)(\pi-2) / \pi^{2}$. Then from (15) and (20) together with the monotonicity of $g(x)$, we clearly see that $f_{u, \alpha}(x)$ is strictly decreasing on $(0,1)$. Therefore, $f_{u, \alpha}(x)<0$ for all $x \in(0,1)$ follows from (13) and the monotonicity of $f_{u, \alpha}(x)$.

Case 3. $2(1-\alpha)(\pi-2) / \pi^{2}<u \leq(1-\alpha)(4 / \pi-1)$. Then (14) leads to

$$
f_{u, \alpha}\left(1^{-}\right) \leq 0
$$

From (15), (19), and (20) together with the monotonicity of $g(x)$, we clearly see that there exists unique $x_{0} \in(0,1)$ such that $f_{u, \alpha}(x)$ is strictly decreasing on $\left(0, x_{0}\right]$ and strictly increasing on $\left[x_{0}, 1\right)$. Therefore, $f_{u, \alpha}(x)<0$ for all $x \in$ $(0,1)$ follows from (13) and (21) together with the piecewise monotonicity of $f_{u, \alpha}(x)$.

Case 4. $(1-\alpha)(4 / \pi-1)<u \leq(1-\alpha) / 3$. Then (14) leads to

$$
f_{u, \alpha}\left(1^{-}\right)>0
$$

It follows from (15), (19), and (20) together with the monotonicity of $g(x)$, there exists unique $x_{1} \in(0,1)$ such that $f_{u, \alpha}(x)$ is strictly decreasing on $\left(0, x_{1}\right]$ and strictly increasing on $\left[x_{1}, 1\right)$. Equation (13) and inequality (22) together with the piecewise monotonicity of $f_{u, \alpha}(x)$ lead to the conclusion that there exists $x_{2} \in\left(x_{1}, 1\right)$ such that $f_{u, \alpha}(x)<0$ for $x \in\left(0, x_{2}\right)$ and $f_{u, \alpha}(x)>0$ for $x \in\left(x_{2}, 1\right)$.

Lemma 3. Let $\lambda, \alpha \in(0,1)$ and

$$
\varphi_{\lambda, \alpha}(x)=\lambda x^{2}-(1-\alpha)\left(\frac{x}{\sinh ^{-1}(x)}-1\right) .
$$

Then $\varphi_{\lambda, \alpha}(x)>0$ for all $x \in(0,1)$ if and only if $\lambda \geq(1-\alpha) / 6$ and $\varphi_{\lambda, \alpha}(x)<0$ for all $x \in(0,1)$ if and only if $\lambda \leq(1-\alpha)(1-$ $\log (1+\sqrt{2})) / \log (1+\sqrt{2})$.

Proof. From (23) we get

$$
\begin{gathered}
\varphi_{\lambda, \alpha}\left(0^{+}\right)=0, \\
\varphi_{\lambda, \alpha}\left(1^{-}\right)=\lambda-\frac{(1-\alpha)[1-\log (1+\sqrt{2})]}{\log (1+\sqrt{2})}, \\
\varphi_{\lambda, \alpha}^{\prime}(x)=2 x\left[\lambda-\frac{1-\alpha}{2} \psi(x)\right],
\end{gathered}
$$

where

$$
\psi(x)=\frac{\sinh ^{-1}(x)-x / \sqrt{1+x^{2}}}{x\left(\sinh ^{-1}(x)\right)^{2}} .
$$

Let $\psi_{1}(x)=\sinh ^{-1}(x)-x / \sqrt{1+x^{2}}$ and $\psi_{2}(x)=$ $x\left(\sinh ^{-1}(x)\right)^{2}$, then

$$
\psi(x)=\frac{\psi_{1}(x)}{\psi_{2}(x)}, \quad \psi_{1}(0)=\psi_{2}(0)=0
$$




$$
\begin{aligned}
& \frac{\psi_{1}^{\prime}(x)}{\psi_{2}^{\prime}(x)} \\
& =x^{2} \times\left(\left(1+x^{2}\right)^{3 / 2}\left(\sinh ^{-1}(x)\right)^{2}\right. \\
& \left.\quad+2 x\left(1+x^{2}\right) \sinh ^{-1}(x)\right)^{-1} \\
& =\left(\left(\left(1+x^{2}\right)^{3 / 4} \sinh ^{-1}(x) / x\right)^{2}\right. \\
& \left.\quad+2\left(1+x^{2}\right)^{1 / 4}\left(\left(1+x^{2}\right)^{3 / 4} \sinh ^{-1}(x) / x\right)\right)^{-1} .
\end{aligned}
$$

It is not difficult to verify that the function $\left(1+x^{2}\right)^{3 / 4}$ $\sinh ^{-1}(x) / x$ is strictly increasing on $(0,1)$. Then $(28)$ together with Lemma 1 leads to the conclusion that $\psi(x)$ is strictly decreasing on $(0,1)$. Moreover, making use of L'Hôpital's rule, we have

$$
\begin{gathered}
\psi\left(0^{+}\right)=\frac{1}{3}, \\
\psi\left(1^{-}\right)=\frac{\sqrt{2} \log (1+\sqrt{2})-1}{\sqrt{2} \log ^{2}(1+\sqrt{2})} .
\end{gathered}
$$

We divide the proof into four cases.

Case 1. $\lambda \geq(1-\alpha) / 6$. Then from (26) and (29) together with the monotonicity of $\psi(x)$, we clearly see that $\varphi_{\lambda, \alpha}(x)$ is strictly increasing on $(0,1)$. Therefore, $\varphi_{\lambda, \alpha}(x)>0$ for all $x \in(0,1)$ follows from (24) and the monotonicity of $\varphi_{\lambda, \alpha}(x)$.

Case 2. $\lambda \leq(1-\alpha)[\sqrt{2} \log (1+\sqrt{2})-1] /\left[2 \sqrt{2} \log ^{2}(1+\sqrt{2})\right]$. Then from (26) and (30) together with the monotonicity of $\psi(x)$, we clearly see that $\varphi_{\lambda, \alpha}(x)$ is strictly decreasing on $(0,1)$. Therefore, $\varphi_{\lambda, \alpha}(x)<0$ for all $x \in(0,1)$ follows from $(24)$ and the monotonicity of $\varphi_{\lambda, \alpha}(x)$.

Case 3. $\left((1-\alpha)[\sqrt{2} \log (1+\sqrt{2})-1] / 2 \sqrt{2} \log ^{2}(1+\sqrt{2})\right)<\lambda \leq$ $((1-\alpha)[1-\log (1+\sqrt{2})] / \log (1+\sqrt{2}))$. Then (25) leads to

$$
\varphi_{\lambda, \alpha}\left(1^{-}\right) \leq 0 \text {. }
$$

From (26), (29), and (30) together with the monotonicity of $\psi(x)$, we clearly see that there exists $x_{3} \in(0,1)$ such that $\varphi_{\lambda, \alpha}(x)$ is strictly decreasing on $\left(0, x_{3}\right]$ and strictly increasing on $\left[x_{3}, 1\right)$. Therefore, $\varphi_{\lambda, \alpha}(x)<0$ for all $x \in(0,1)$ follows from (24) and (31) together with the piecewise monotonicity of $\varphi_{\lambda, \alpha}(x)$.

Case 4. $((1-\alpha)[1-\log (1+\sqrt{2})] / \log (1+\sqrt{2}))<\lambda<((1-\alpha) / 6)$. Then (25) leads to

$$
\varphi_{\lambda, \alpha}\left(1^{-}\right)>0 .
$$

It follows from (26), (29), and (30) together with the monotonicity of $\psi(x)$, there exists $x_{4} \in(0,1)$ such that $\varphi_{\lambda, \alpha}(x)$ is strictly decreasing on $\left(0, x_{4}\right]$ and strictly increasing on $\left[x_{4}, 1\right)$. Equation (24) and inequality (32) together with the piecewise monotonicity of $\varphi_{\lambda, \alpha}(x)$ lead to the conclusion that there exists $x_{5} \in\left(x_{4}, 1\right)$ such that $\varphi_{\lambda, \alpha}(x)<0$ for $x \in\left(0, x_{5}\right)$ and $\varphi_{\lambda, \alpha}(x)>0$ for $x \in\left(x_{5}, 1\right)$.

\section{Main Results}

Theorem 4. If $\alpha \in(0,1)$ and $r_{1}, s_{1} \in(1 / 2,1)$, then the double inequality

$$
\begin{aligned}
& C\left(r_{1} a+\left(1-r_{1}\right) b, r_{1} b+\left(1-r_{1}\right) a\right) \\
& <\alpha A(a, b)+(1-\alpha) T(a, b) \\
& <C\left(s_{1} a+\left(1-s_{1}\right) b, s_{1} b+\left(1-s_{1}\right) a\right)
\end{aligned}
$$

holds for all $a, b>0$ with $a \neq b$ if and only if $r_{1} \leq[1+$ $\sqrt{(1-\alpha)(4-\pi) / \pi}] / 2$ and $s_{1} \geq[1+\sqrt{(1-\alpha) / 3}] / 2$.

Proof. Since $A(a, b), T(a, b)$, and $C(a, b)$ are symmetric and homogeneous of degree one, without loss of generality, we assume that $a>b$. Let $p \in(1 / 2,1)$ and $x=(a-b) /(a+b)$, then $x \in(0,1)$ and

$$
\begin{aligned}
& C(p a+(1-p) b, p b+(1-p) a) \\
& \quad-[\alpha A(a, b)+(1-\alpha) T(a, b)] \\
& \quad=A(a, b)\left[(2 p-1)^{2} x^{2}-(1-\alpha)\left(\frac{x}{\arctan x}-1\right)\right] .
\end{aligned}
$$

Therefore, Theorem 4 follows easily from Lemma 2 and (34).

Theorem 5. If $\alpha \in(0,1)$ and $r_{2}, s_{2} \in(1 / 2,1)$, then the double inequality

$$
\begin{aligned}
& C\left(r_{2} a+\left(1-r_{2}\right) b, r_{2} b+\left(1-r_{2}\right) a\right) \\
& \quad<\alpha A(a, b)+(1-\alpha) M(a, b) \\
& \quad<C\left(s_{2} a+\left(1-s_{2}\right) b, s_{2} b+\left(1-s_{2}\right) a\right)
\end{aligned}
$$

holds for all $a, b>0$ with $a \neq b$ if and only if $s_{2} \geq[1+\sqrt{(1-\alpha) / 6}] / 2$ and $r_{2} \leq[1+$ $\sqrt{(1-\alpha)(1-\log (1+\sqrt{2})) / \log (1+\sqrt{2})}] / 2$.

Proof. Since $A(a, b), M(a, b)$, and $C(a, b)$ are symmetric and homogeneous of degree one, without loss of generality, we assume that $a>b$. Let $q \in(1 / 2,1)$ and $x=(a-b) /(a+b)$, then $x \in(0,1)$ and

$$
\begin{aligned}
C & (q a+(1-q) b, q b+(1-q) a) \\
& -[\alpha A(a, b)+(1-\alpha) M(a, b)] \\
& =A(a, b)\left[(2 q-1)^{2} x^{2}-(1-\alpha)\left(\frac{x}{\sinh ^{-1}(x)}-1\right)\right] .
\end{aligned}
$$

Therefore, Theorem 5 follows easily from Lemma 3 and (36). 
Remark 6. If $\alpha=0$, then Theorem 4 reduces to the first double inequality in (8).

Corollary 7. If $\lambda, \mu \in(1 / 2,1)$, then the double inequality

$$
\begin{aligned}
& C(\lambda a+(1-\lambda) b, \lambda b+(1-\lambda) a) \\
& \quad<M(a, b)<C(\mu a+(1-\mu) b, \mu b+(1-\mu) a)
\end{aligned}
$$

holds for all $a, b>0$ with $a \neq b$ if and only if $\lambda \leq[1+$ $\sqrt{1 / \log (1+\sqrt{2})-1}] / 2$ and $\mu \geq(6+\sqrt{6}) / 12$.

Proof. Corollary 7 follows easily from Theorem 5 with $\alpha=$ 0 .

\section{Acknowledgments}

This research was supported by the Natural Science Foundation of China (Grants no.11171307, 61173123), the Natural Science Foundation of Zhejiang Province (Grants no. Z1110551, LY12F02012), and the Natural science Foundation of Huzhou City (Grant no. 2012YZ06).

\section{References}

[1] E. Neuman and J. Sándor, "On the Schwab-Borchardt mean," Mathematica Pannonica, vol. 14, no. 2, pp. 253-266, 2003.

[2] H.-J. Seiffert, “Aufgabe $\beta$ 16," Die Wurzel, vol. 29, pp. 221-222, 1995.

[3] Y.-M. Chu and B.-Y. Long, "Bounds of the Neuman-Sándor mean using power and identric means," Abstract and Applied Analysis, vol. 2012, Article ID 832591, 6 pages, 2012.

[4] Y.-M. Chu, B.-Y. Long, W.-M. Gong, and Y.-Q. Song, "Sharp bounds for Seiffert and Neuman-Sándor means in terms of generalized logarithmic means," Journal of Inequalities and Applications, vol. 2013, article 10, 2013.

[5] F. R. Villatoro, "Local error analysis of Evans-Sanugi, nonlinear one-step methods based on $\theta$-means," International Journal of Computer Mathematics, vol. 87, no. 5, pp. 1009-1022, 2010.

[6] F. R. Villatoro, "Stability by order stars for non-linear thetamethods based on means," International Journal of Computer Mathematics, vol. 87, no. 1-3, pp. 226-242, 2010.

[7] J. Pahikkala, "On contraharmonic mean and Phythagorean triples," Elemente der Mathematik, vol. 65, no. 2, pp. 62-67, 2010.

[8] O. Y. Ababneh and R. Rozita, "New third order Runge Kutta based on contraharmonic mean for stiff problems," Applied Mathematical Sciences, vol. 3, no. 5-8, pp. 365-376, 2009.

[9] S. Toader and G. Toader, "Complementaries of Greek means with respect to Gini means," International Journal of Applied Mathematics \& Statistics, vol. 11, no. N07, pp. 187-192, 2007.

[10] Y. Lim, "The inverse mean problem of geometric and contraharmonic means," Linear Algebra and Its Applications, vol. 408, pp. 221-229, 2005.

[11] S. Toader and G. Toader, "Generalized complementaries of Greek means," Pure Mathematics and Applications, vol. 15, no. 2-3, pp. 335-342, 2004.

[12] Y.-M. Chu, M.-K. Wang, and Y.-F. Qiu, "Optimal Lehmer mean bounds for the geometric and arithmetic combinations of arithmetic and Seiffert means," Azerbaijan Journal of Mathematics, vol. 2, no. 1, pp. 3-9, 2012.
[13] P. A. Hästö, "A monotonicity property of ratios of symmetric homogeneous means," JIPAM, vol. 3, no. 5, article 71, 2002.

[14] Y.-M. Chu, M.-K. Wang, and Y.-F. Qiu, "An optimal double inequality between power-type Heron and Seiffert means," Journal of Inequalities and Applications, vol. 2010, Article ID 146945, 11 pages, 2010.

[15] M.-K. Wang, Y.-F. Qiu, and Y.-M. Chu, "Sharp bounds for Seiffert means in terms of Lehmer means," Journal of Mathematical Inequalities, vol. 4, no. 4, pp. 581-586, 2010.

[16] E. Neuman and J. Sándor, "On the Schwab-Borchardt mean. II," Mathematica Pannonica, vol. 17, no. 1, pp. 49-59, 2006.

[17] Y.-M. Li, B.-Y. Long, and Y.-M. Chu, "Sharp bounds for the Neuman-Sándor mean in terms of generalized logarithmic mean," Journal of Mathematical Inequalities, vol. 6, no. 4, pp. 567-577, 2012.

[18] E. Neuman, "A note on a certain bivariate mean," Journal of Mathematical Inequalities, vol. 6, no. 4, pp. 637-643, 2012.

[19] T.-H. Zhao, Y.-M. Chu, and B.-Y. Liu, "Optimal bounds for Neuman-Sándor mean in terms of the convex combinations of harmonic, geometric, quadratic, and contraharmonic means," Abstract and Applied Analysis, vol. 2012, Article ID 302635, 9 pages, 2012.

[20] Y.-M. Chu, C. Zong, and G.-D. Wang, "Optimal convex combination bounds of Seiffert and geometric means for the arithmetic mean," Journal of Mathematical Inequalities, vol. 5, no. 3, pp. 429-434, 2011.

[21] Y.-M. Chu, M.-K. Wang, and W.-M. Gong, "Two sharp double inequalities for Seiffert mean," Journal of Inequalities and Applications, vol. 2011, article 44, 2011.

[22] Y.-M. Chu and S.-W. Hou, "Sharp bounds for Seiffert mean in terms of contraharmonic mean," Abstract and Applied Analysis, vol. 2012, Article ID 425175, 6 pages, 2012.

[23] Y.-M. Chu, S.-W. Hou, and Z.-H. Shen, "Sharp bounds for Seiffert mean in terms of root mean square," Journal of Inequalities and Applications, vol. 2012, article 11, 2012.

[24] G. D. Anderson, M. K. Vamanamurthy, and M. K. Vuorinen, Conformal Invariants, Inequalities, and Quasiconformal Maps, Canadian Mathematical Society Series of Monographs and Advanced Texts, John Wiley \& Sons, New York, NY, USA, 1997. 


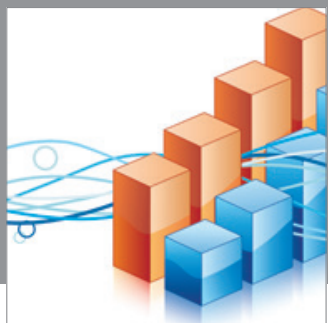

Advances in

Operations Research

mansans

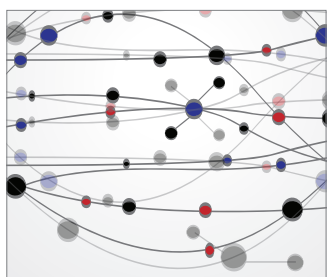

The Scientific World Journal
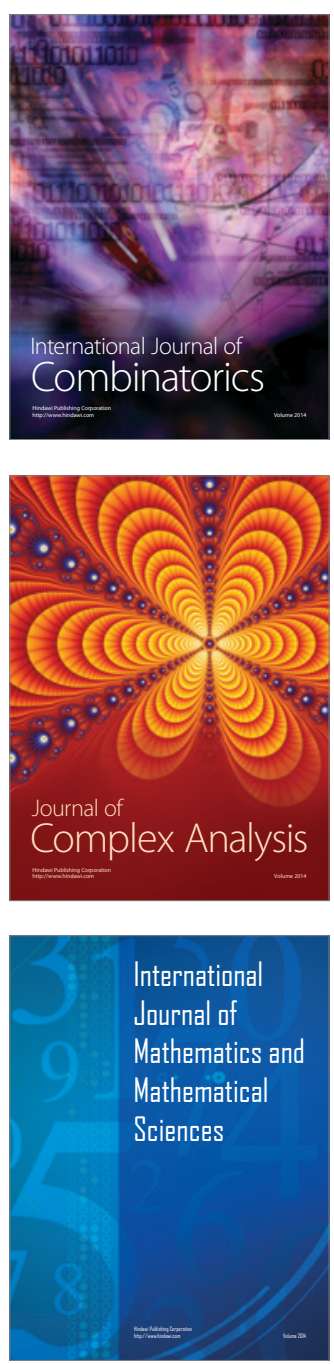
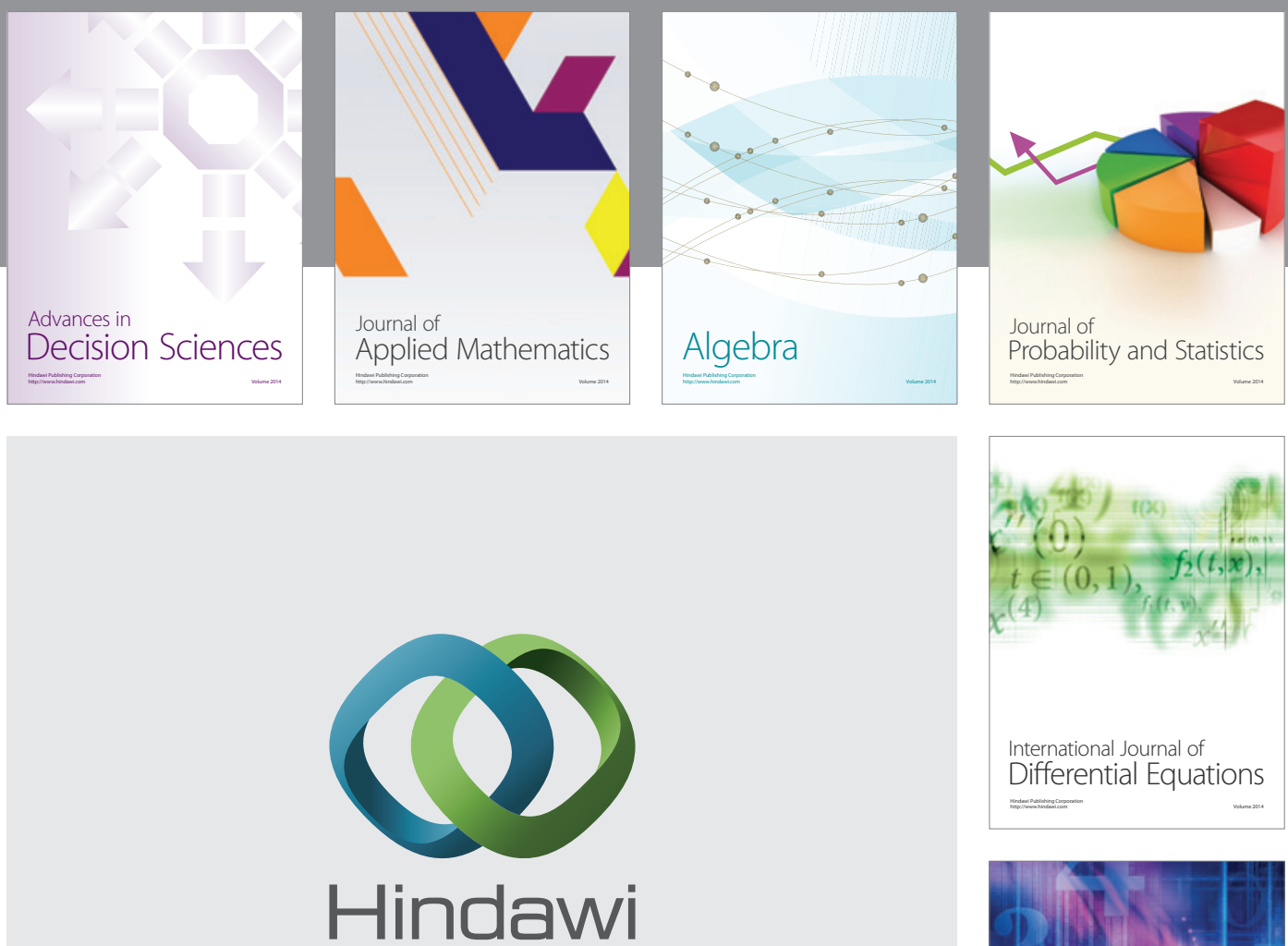

Submit your manuscripts at http://www.hindawi.com
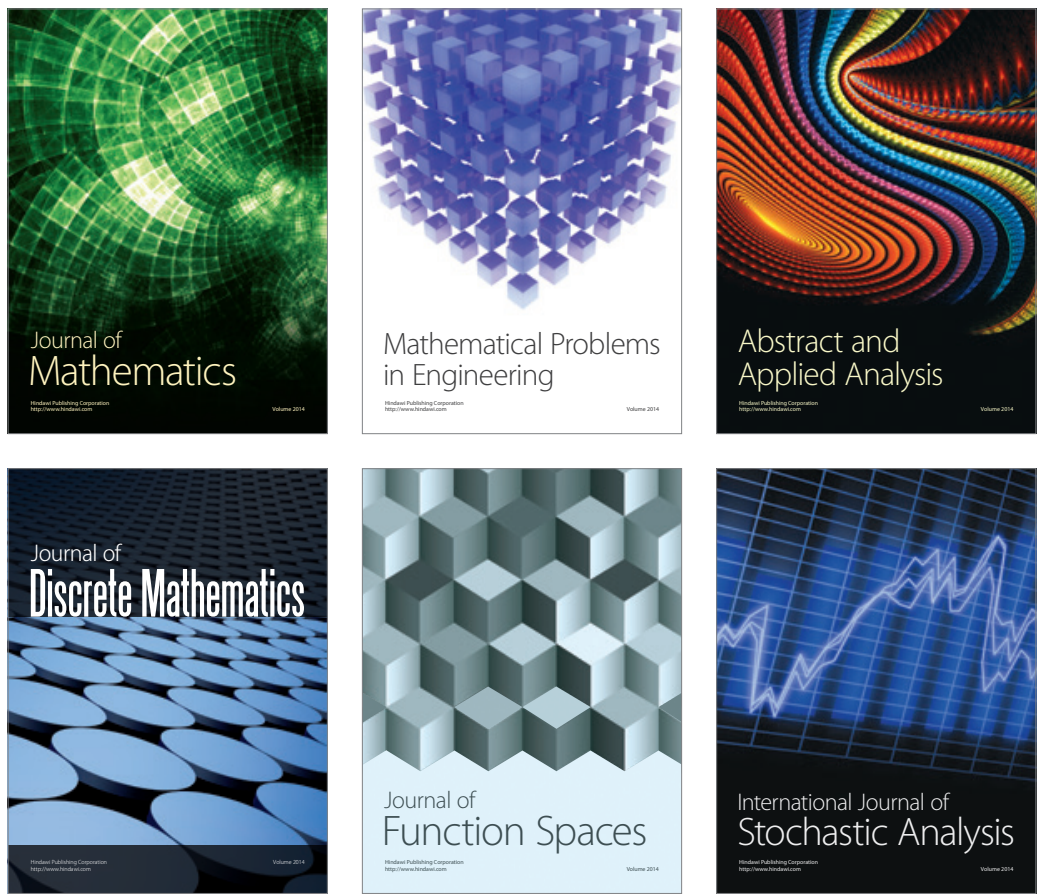

Journal of

Function Spaces

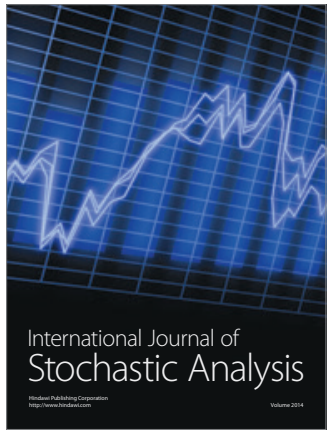

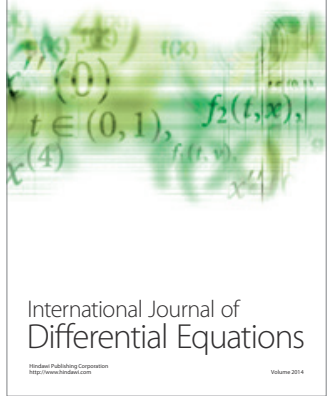
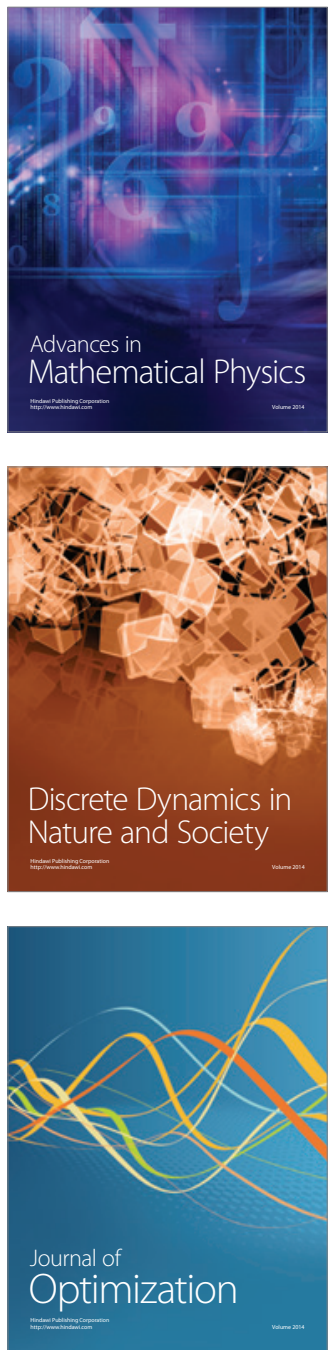